REFERENCES AND/OR ACKNOWLEDGEMENTS

1. Allenet B, et al. Pharm World Sci 2006;28:181-188.

No conflict of interest.

\section{CPS-191 PHARMACEUTICAL INTERVENTIONS IN A MEDICATION RECONCILIATION PROGRAMME ON ADMISSION IN SURGICAL PATIENTS}

L Martinez-Dueñas Lopez-Marin, T Vilchez Medina, CL Muñoz Cid ${ }^{*}$, R Claramunt García, AM Lopez Lopez, E Perez Cano, Y Jimenez Lopez, N Garcia Gomez. Hospital Universitario De Jaén, Pharmacy, Jaén, Spain

\subsection{6/ejhpharm-2020-eahpconf.292}

Background and importance Medication errors at hospital admission are common, increasing morbidity and mortality. The pharmacist can help to prevent the occurrence of medication related problems through medication reconciliation.

Aim and objectives To analyse the pharmaceutical interventions performed during the implementation of a medication reconciliation programme on hospital admission to reduce medication errors (ME).

Material and methods This was an observational prospective study (October 2018-September 2019). Patients older than 65 years who received at least five drugs and had more than 24 hours of admission in the general surgery and urology units were included. Variables considered were age, sex, number of prescribed drugs and ME. The best pharmacotherapeutic history was developed, including diagnosis, medical history and complete list of chronic home medication, consulting the electronic history programme of electronic prescriptions. This information was completed with an interview with the patient/ caregiver. In the event of any discrepancy, the responsible doctor was contacted.

Results Medication reconciliation was conducted for 553 patients. Median age was 75 years and $56.6 \%$ were men. The average number of medications per patient at admission was 8.2. A total of 4567 drugs were reconciled, with a total of 2404 interventions in the discrepancies found: 1586 (65.9\%) were justified while $818(34.1 \%)$ were classified as unjustified or ME (omission (90.17\%), dose (2.7\%), frequency, schedule or route of administration (1.69\%), therapeutic duplicity (1\%) and other), with a degree of acceptance of $62 \%$, correcting the discrepancy in most cases before 24 hours had elapsed. Communication with the doctor was done by electronic messaging in $91 \%$ of cases.

Conclusion and relevance We observed that during the medication reconciliation, numerous $\mathrm{ME}$ were detected, the majority of which were omission of medications. The involvement of the pharmacist, integrated into a multidisciplinary team together with doctors and nurses, allowed the detection of discrepancies, obtaining a high percentage of acceptance of the interventions, thus reducing ME. The medication reconciliation programmes allow the detection and resolution of discrepancies, preventing $\mathrm{ME}$ in healthcare transitions.

\section{REFERENCES AND/OR ACKNOWLEDGEMENTS}

No conflict of interest.

\section{CPS-192 ESTIMATING THE SURVIVAL PROGNOSIS OF PATIENTS WITH ADVANCED GASTROINTESTINAL MALIGNANCY ON HOME PARENTERAL NUTRITION: A RETROSPECTIVE, MONOCENTRE STUDY}

J Neefs*, I Spriet, L De Pourcq, P Declercq. University Hospitals Leuven, Hospital Pharmacy, Leuven, Belgium

\subsection{6/ejhpharm-2020-eahpconf.293}

Background and importance The initiation of home parenteral nutrition (HPN) in patients with advanced malignancy is a highly controversial topic. Guidelines recommend reserving this therapy for patients with an expected survival of longer than 2-3 months. Administering HPN in patients with a shorter survival probably has little benefit, while creating the risk of PN related complications. As HPN in advanced cancer patients is becoming increasingly common in our hospital, we wanted to investigate whether current practices are supported by the rational use of HPN.

Aim and objectives Firstly, this study sought to investigate the proportion of patients with advanced cancer receiving HPN in our hospital, surviving for longer than 2-3 months. Furthermore, we wanted to investigate whether the application of survival prediction models could improve estimation of the length of patient survival.

Material and methods Survival proportions of 250 patients with advanced gastrointestinal malignancy receiving HPN in our hospital during 2008-2016 were examined. Additionally, agreement was assessed between observed survival times and the current inhospital survival prediction method (ie, physician's clinical judgement) or survival estimation by a published prediction nomogram. Moreover, through the use of multivariable logistic regression on variables gathered from the studied patient set, both a 2 and 3 month survival prediction model were constructed and validated.

Results The results showed that a relatively low proportion of patients actually met the proposed survival criteria (65.2\% and $46.4 \%$ for 2 and 3 month survival lengths, respectively). Concerning survival prediction, clinicians predominantly tended to overestimate survival length. Furthermore, application of the published nomogram did not improve survival prediction. Therefore, de novo 2 and 3 month survival prediction models were developed. The 2 month prediction model consisted of four variables: Karnofsky performance score (KPS), Glasgow prognostic score (GPS), gender and serum sodium, while the 3 month model consisted of three variables: KPS, GPS and serum urea. Validation of constructed survival prediction models in an independent set of 99 patients showed discriminatory abilities that were comparable, but not superior, to the results obtained with the aforementioned survival prediction nomogram.

Conclusion and relevance This investigation showed that correct patient survival prediction remains an intrinsically difficult exercise. In order for our constructed models to have clinical utility, further improvement is needed, possibly through the inclusion of additional predictors for survival.

\section{REFERENCES AND/OR ACKNOWLEDGEMENTS}

No conflict of interest. 